\title{
Graphic and cultural aspects of pictograms: an information ergonomics viewpoint
}

\author{
Carla Galvão Spinillo* \\ Department of Design, The Federal University of Paraná, Rua Gal. Carneiro, 460, Edf. D. Pedro I Sala 811, \\ Centro, Curitiba, PR, Brazil, 80060-050
}

\begin{abstract}
The use of pictograms is discussed considering their information content, graphic complexity and cultural dimension. The resemblance and the illusion theories are highlighted to define pictogram as a salience-based representation system, which communicational efficacy depends upon historical and cultural aspects in their interpretation. Thus, the competence in interpreting pictograms is considered relative to users' acquaintance with the pictorial system and with the referents. Pictogram as a general/neutral visual statement is questioned, pointing out the cultural and gender attributes added to pictures to represent people, professions and social events. As a result of this discussion, some critical points of the standardization of pictograms are presented.
\end{abstract}

Keywords: pictogram, graphic aspects, cultural aspects, information ergonomics

\section{Introduction}

Pictograms are considered graphic representations of concepts through visual synthesis, used to communicate messages to broad audiences $[1,2]$. They are for public information, and generally designed to be effective without the use of words, and to be read in a prompt manner [1]. Pictograms are also considered to present advantages over text/words in communicating messages, as they can be seen from distance, mainly in adverse visual conditions (e.g., fog in roads), they can be understood by people differing in language skills/literacy, and they can represent information in a compact manner in condensed areas, i.e., the visual property of compactness [1].

Several authors have discussed the pictograms whether regarding their components, functions or communication effectiveness [1, 3, 4, 5]. Moreover, taxonomies and guidelines have been proposed to improve the way pictograms can be employed to convey information to audiences $[1,2]$. Despite the relevance of these authors' contributions to the design of pictograms, some questions remain to be answered regarding how do we represent and perceive pictograms. This papers attempts to fill this gap by relating theories of depiction and perception of images to pictograms, together with the cultural aspects that may affect their communication effectiveness. Thus, initially the syntactic and semantic aspects of pictorial representation are presented to set the ground. Then, the resemblance and the illusion theories are highlighted $[6,7]$, followed by the discussion of generative and transferable competence in perceiving/interpreting pictures [8]. Finally, cultural aspects are discussed as they may affect comprehension of pictograms.

\section{Syntactic and semantic aspects of pictograms}

The syntactic aspects of pictograms regard the pictorial and verbal components, their visual attributes and relations. Looking at the number of pictorial components, a pictogram can be composed by a single element (simple unit) or by combined elements (composite unit). Composite unit is common in pictograms to represent complex information/statement, such as in the warning of an airplane safety instruction leaflet that states: "if you see fire or smoke through the window, do not open the emergency door' (Figure 1a). However, how effective this picto-

\footnotetext{
* Corresponding author. cgspin@gmail.com
} 
gram is in communicating the message can be questioned, as it demands readers/passengers to interpret several elements in an integrated manner as a visual narrative, to say the least. In relation to the verbal components of a pictogram, they are presented as labels to the images. Nevertheless, some pictograms present letters to convey an abstract concept, such as the lower case ' i' for 'touristic information' (Figure 1b). In such cases, the letters have the role of 'image' in the pictogram. Again, the communication effectiveness of this kind of pictograms can be questioned, as their interpretation rely on people's previous knowledge of the representation.

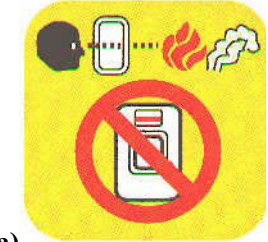

(a)

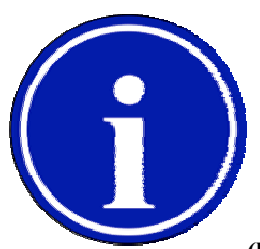

(b)
Figure 1: Examples of pictograms (composite and simple units). Source: Author's collection

It is worth highlighting that the pictorial and verbal modes differ in communicational competence. Pictures are more appropriate than words to represent material world/existing things, relative size and simultaneous concepts. Whereas texts/words are more appropriate to represent general and abstract concepts. The pictorial and verbal modes of representation have distinct and equally important roles in communicating a message in pictograms. Their efficacy depends upon the kind of information to be represented (e.g. abstract or existing thing) allied to constraints posed by readers' domain (e.g. level of literacy, picture familiarity). In this sense, the warning in Figure 1a is an example of the limitations of pictures to represent conditional situations. The conditions IF and OR ('IF you see fire OR smoke through the window...') are implied in the pictogram, therefore are not obvious to readers.

Pictograms also possess visual attributes that are intended to promote accuracy in the representation of referents or concepts, as well as to easy the visualization of elements.

Horn [9] identified visual attributes to pictorial representations that were based upon Bertin's [10] graphic variables used to analyze cartographic representations. They are: value, texture, color, orientation, size, movement, location (2d and 3D space), thickness and illumination. In the pictogram for disable (wheelchair person) in Figure 2a different line thickness is employed to represent the person and to outline the image which is oriented left to right (facing right), and it is a bi-dimensional drawing. Figure $2 b$ presents a pictogram with similar visual attributes, however adding color (black, blue and red) to distinguish the elements (airplane, water and frame); and the pictogram in Figure 2c employs a tri-dimensional representation to convey the message of 'store the medication under refrigeration'.

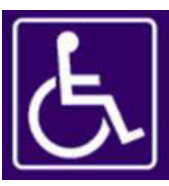

(a)

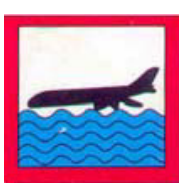

(b)

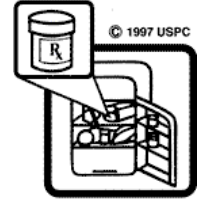

(c)
Figure 2: Examples of visual attributes in pictograms. Source: (a) and (b) Author's collection; and (c) USP (1998)

Regarding the visual relations, pictograms as part of graphic language, constitute a system of interconnected signs, which can be only combined in certain ways to communicate a message. For instance, the semantic marks for prohibition (cross and diagonal bar) are to be positioned over an image, otherwise the concept of prohibition will not be properly conveyed, as shown in Figure 3 (No Parking).
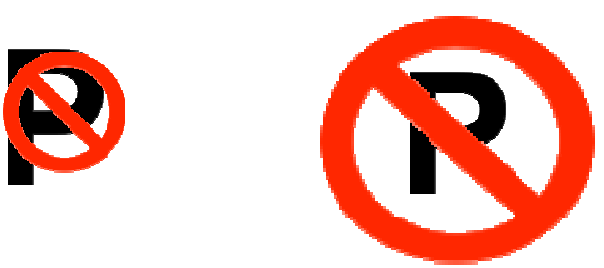

Figure 3: Example of the use of semantic marks. Source: Author's design.

Accordingly, it can be said that the syntactic aspects of pictograms are tightly related to their semantic aspects. These regard the information represented and the relation between the pictorial and verbal modes in pictograms. It is worth pointing out that pictograms can convey affirmative or negative/prohibitive messages (warnings). For the latter, semantic marks for negation are used (abovementioned). However, they may obstruct the visualization of the image, affecting the pictogram legibility. Figure 4a shows a pictogram representing a prohibition, in which the cross in red over the image jeopardizes the visualization of the pictorial elements. 
The semantic mark (diagonal) over the image of a cigarette also obstructs visualization in the pictogram showed in Figure 4b, but in this case due to lacking visual distinction between the diagonal bar and the image, as they seem to be a single element/unit. This ratifies that the success of a pictogram in conveying meaning depends upon the satisfactory integration between syntactic and semantic aspects of the representation.

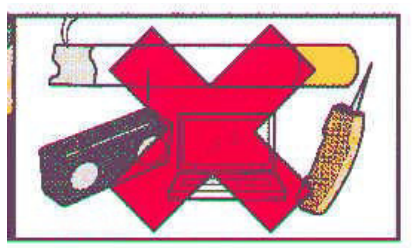

(a)

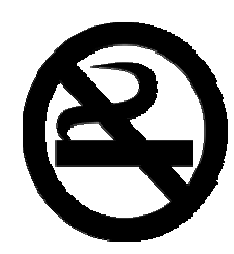

(b)
Figure 4: Examples of visual obstruction in pictograms. Source: Author's collection.

Regarding the semantic relations between image and words in pictograms, they can be of relay, in which pictorial and verbal modes are in a complementary relation; and/or of anchorage, in which the verbal mode supports the meaning of the pictorial mode and vice-versa (same information). For instance, in a pictogram for 'do not smoke' (a cigarette with a cross over it), text and picture are in an anchorage relation when the text says 'do not smoke'. If it says 'do not smoke in the lavatory' text and picture would be in a relay relation, as the text complements the meaning of the image. The relations of anchorage and relay may occur simultaneously in pictograms. However, they may differ in effectiveness in communicating messages, since words/texts may not allow prompt visualization, or at least not as good as images. Thus, the relay relation between image and words/text in pictograms may not be as successful as the anchorage relation.

The semantic relations in pictograms, nevertheless, may not be restricted to the pictorial and verbal modes. They may also regard relations between the concept/referent and its representation, which is discussed next.

\section{The pictorial representation in pictograms}

According to Dewar [1] the relation between the concept/referent in a pictogram and its representation is referred to as 'mapping relation', and can be of: part-part relation; part-whole relation; image-based relation; example-based relation and concept-based relation. In the former (part-part) pictograms share certain characteristics with their referents, as for example a computer icon 'open a paper file' to convey 'access digital information'. In the part- whole relation, a particular characteristic of the referent is used to represent the concept in a pictogram (e.g. envelop representing 'mail'). In the image-based relation, the pictogram is the image of the referent, whereas in the example-based relation, a class of objects is represented by one of its items, as for example a book to represent 'library'. Finally, in the conceptbased relation, an arbitrary image is employed to represent a concept which has no referent or class of referents, such as the pictogram for 'radiation'. Although this is an interesting approach, it seems to not account for the perceptual aspects involved in the representation of messages in pictograms.

Since pictograms can be considered conceptual as well as perceptual representations, their effectiveness depends not only upon readers' interpretation, but also upon readers' perception of what is depicted. In this sense, theories of visual perception and representation may contribute to a better understanding of this theme $[11,7,8,6]$. For the purpose of this paper, two theories that deal with the representation and perception of pictures in a similar way are considered here: the resemblance theory $[6,11]$ and the illusion theory $[11,7]$.

The resemblance theory considers that an image is alike its referent (theme or object) and there is a subjective similarity between the depiction and the perception. The perception of a representation is determined by reader's visual experience with the image and with the referent. Moreover, reader's visual experience with the material world constructs mental sets and cognitive schemas of the world, which allow for the pictorial identification by the reader. Thus, a pictogram resembles its referent through a representational system which is part of reader's cognitive schema or mental set. Figure 5 illustrates that our visual experience with 'ships' allow us to recognize a pictogram for ship/port.

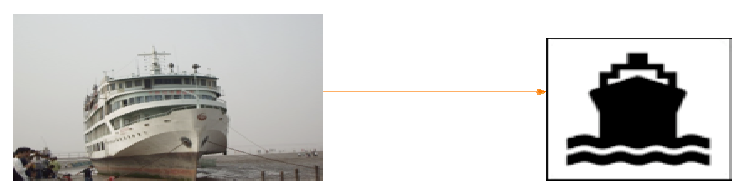

Figure 5: Visual relation between referent and pictogram. Source: Author's collection. 
Now, the illusion theory considers that a picture is an 'illusion' of its referent, in which the co-relation between the depiction and its referent is relatively arbitrary $[11,7]$. Therefore, a pictogram even when depicting a real object is to a certain extent arbitrary. According to this theory, we construct picture schemas which allow/aid us to perceive and represent the material world. All pictures belong to representational systems defined by a cluster of visual saliences which are influenced by readers' pictorial experience, within historical and cultural dimensions. Thus, by considering the resemblance and illusion theories, we can state that: Pictograms are a salience-based representation system, which communicational efficacy depends upon cultural aspects in their interpretation. We perceive and represent pictograms according to our pictorial schematas by using visual synthesis in their design, which should 'match' with our mental set and cognitive schemas. This makes the identification/recognition and interpretation of pictograms possible, regardless the changes a referent may suffer over time and/or due to technological progress. An example of this is the representation of 'telephone' in pictograms, as shown in Figure 6.
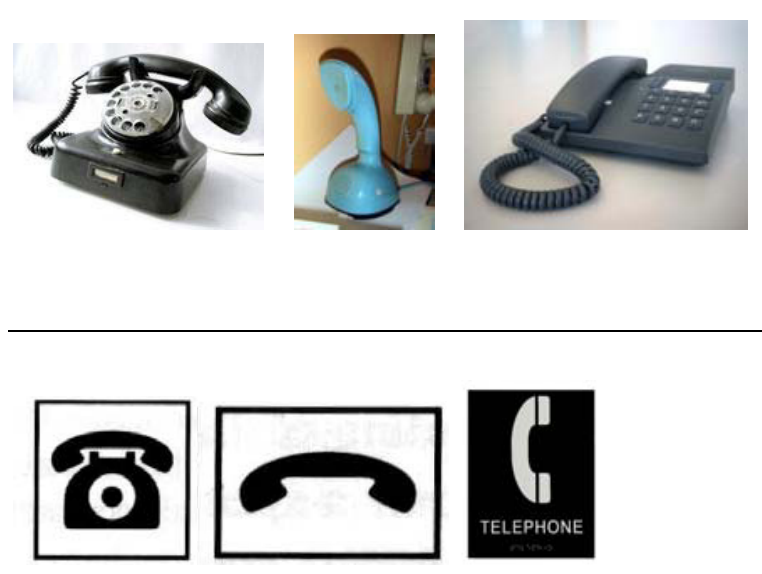

Figure 6: Example of representation of telephones. Source: Authors' collection and designs.

Other aspect to be taken into account is the readers' representational competence for pictograms, in terms of their perception and interpretation of images. Our competence in perceiving and interpreting pictures is generative and transferable [8]. The ability of interpreting certain pictures generates the ability of interpreting other pictures as far as we know what the referent depicted is like. This is referred to as the pictorial generative competence. Accordingly, a per- son who is able to interpret some pictograms would also be able to interpret any pictogram of a familiar referent. In addition, readers' pictorial competence may also be transferable. Knowing a pictorial system leads to understand the possible representations within the system. In this sense, a person who is acquainted with the visual synthesis employed in pictograms in his/her town will also be able to interpret pictograms in any town as far as they present similar visual synthesis. Thus, readers' competence in interpreting pictograms is related to their acquaintance with pictorial systems, which enable them to perceive and interpret a depiction according to their degree of familiarity with its referent and with the system.

Nevertheless, the understanding and the acceptability of pictograms may be affected by aspects other than perceptual ones. The cultural factor may play a part in this, as discussed next.

\section{The role of culture}

Readers' cultural characteristics, such as values, age, gender and visual literacy, affect the interpretation and even the acceptance of the representation of information in pictograms, as in any visual message [12]. In this regard, different readers/audiences may have different referents for the same concept. For instance, a pictogram representing 'restaurant' for the US audience may depict a fork and knife, whereas for a Chinese audience a picture of a chopstick should be used.

The use of cartoons or humor in the representation of pictograms is other aspect that affects not only their understanding, but also their acceptability by readers [2]. Depending on the cultural (social and religious) values of the intended audience, and of the seriousness of the topic addressed, such representational strategies may lead to failure in communication. For instance, a 'funny' pictogram for 'male toilet' (Figure 7) may be welcomed by young people in night clubs, but possibly not by patients in a hospital.

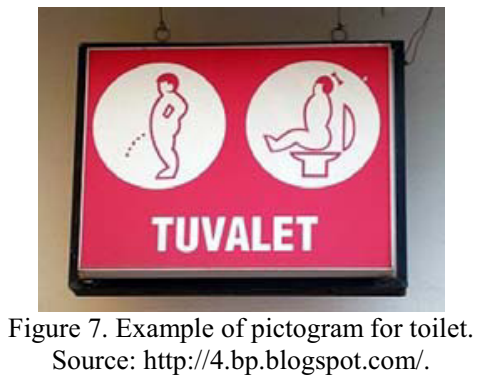


Gender representation is other relevant aspect in the design of pictograms. Pictograms are mostly intended to make general visual statements, seeking 'neutral' representations. However, the pictorial mode of representation is not able to represent general or abstract concepts, as earlier mentioned in this paper. They need words to support them, to put the message across. Thus, neutralization is not an easy goal in pictorial representation, to say the least. As a result, specific gender attributes as well as cultural and racial ones are attached to pictures to represent people, professions and social events. Figure 8 shows pictograms for 'male and female toilets' in different countries (Taiwan, Brazil and India) discussed by Darras [5]. According to the country culture, different visual characteristics regarding the clothing of the man and woman depictions are employed, affecting gender representations, as stressed by the author. If the pictograms designed in Brazil were used in India, they will probably be not effective for the Indian audience.

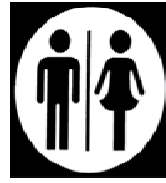

Taiwan

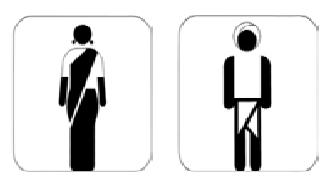

India

Figure 8: Examples of toilet signs in different countries. Source: Darras (2007: 164).

Darras [5] goes further in the discussion of gender in pictograms, asserting that there is a male oriented culture. To support this, some examples of pictograms in several countries are presented, as ones for 'lift/elevator', 'school' and 'family'. The former is in Figure 9 that shows the concept of 'people' represented mainly by male images inside a lift, and in Figure 10 there are examples of pictograms for 'school in which the male image seems to have the main role in the representation. The criticism towards gender in pictograms is intensified when commenting the representation of 'family'. The pictogram depicts (from left to right) a child (son), a man (father), a woman (mother) and a stroller (baby) giving the impression that the man, again, plays the main role (Figure 11). Thus, he comments: 'Men still represent the strength and the protection for the 'weaker sex' and the confinement of women to their role of mother and modest reproducer is still generalized' [5: 165]. Moreover, he questions the impact of these images on the construction of representations of humans and of their social roles.

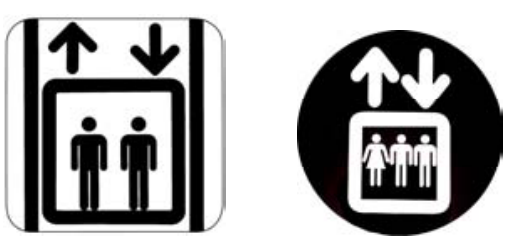

Figure 9: Examples of lift signs. Source: Darras (2007: 159).

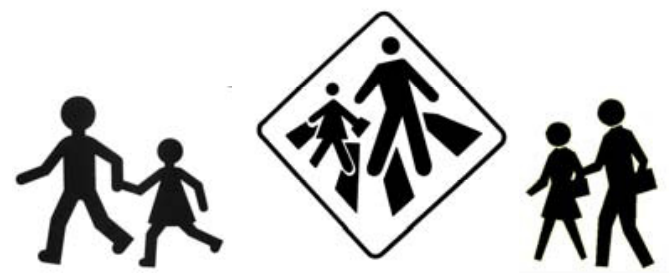

Figure 10: Examples of school signs. Source: Darras (2007: 165).

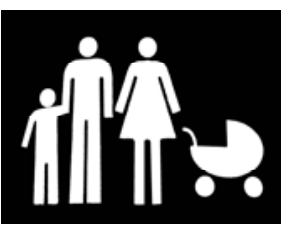

Figure 11: Examples of sign for family. Source: Darras (2007: 165).

Despite the passionate way the author embraces the issue of gender representation in pictograms, one can agree that the male image takes precedence over the female image. And, whether conscious or not, pictograms' designers are promoting a male oriented thinking in the representation of general concepts. Nevertheless, by considering the important role women play in today's society and economy (family and workplace), this is at least an issue that deserves attention of designers to produce representations in tune with current realities 


\section{Final considerations}

Taking into account the aspects discussed here, it is plausible to claim that pictograms are more than 'graphic representations of concepts through visual synthesis used to communicate messages to broad audiences' [1]. They can be regarded as communication artifacts that integrate syntactic and semantic aspects of a message, which are related to readers' perception of pictorial representation and, their production and interpretation are embedded in a cultural context.

Finally, the aspects discussed may also lead to question the standardization of pictograms to the extent an internationalization of visual messages is culturally appropriate and effective $[1,5,13]$. Let us push further by claiming that the design of pictograms should (when possible) be customized by acknowledging the cultural diversity and genders. Thus, pictograms should be designed with an 'accent', a cultural and gender accent.

\section{References}

[6] Arnheim, R. (2000). Arte e Percepção Visual: uma psicologia da visão criadora. 12.ed. São Paulo, SP: Livraria Pioneira Editora.

[2] Azevedo, E. R.; Spinillo, C. G. (2006). Algumas considerações sobre o design de sinais de advertência as em manuais de instrução de fogões. In Anais do 7o P\&D- Congresso Brasileiro de Pesquisa e Desenvolvimento em Design Curitiba, PR: AEND- Associação de Ensino e Pesquisa em Design no Brasil. pp. 124-128.
[10]Bertin, J. (1983). Semiology of graphics: diagrams, networks, maps. Translated by William J. Berg. London: The University of Wisconsin Press

[5] Darras. B. (2007). Semiotic of visual signs and information design. In: C. Spinillo; S. G. Coutinho (eds). Selected Readings of the Information Design International Conference. São Paulo: SBDI- Brazilian Society of Information Design. pp 154-166.

[1] Dewar, R. (1999). Design and evaluation of public information symbols. Visual Information for everyday use: design and research perspectives. London, UK: Taylor \& Francis. pp. 285-304

[11] Gombrich, E. H. (1960). Art and Illusion: a study of the psychology of pictorial representation. New York: Pantheon Books

[7] Gombrich, E. H. (1999). The uses of images: studies in the social function of art and visual communication. London, UK: Phaidon Press.

[9] Horn, R. E. (1998).Visual Language: global communication for the 21 st century. Bainbridge Island, Washington: MacroVU, Inc

[4] Leite, C; Spinillo, C. G.; Soares, M. M. (2007). As contribuições da linguagem gráfica pictórica para o design de avisos e advertências em medicamentos. In: Anais do Congresso Internacional De Pesquisa em Design, 4. Rio de Janeiro. Anais.. Rio de Janeiro: Associação Nacional de Pesquisa em Design. $8 \mathrm{p}$.

[3] Lesch, M. (2006). Consumer product warnings: research and recommendations. In: M.S. Wogalter (Ed). Handbook of warning. Lawrence Erlmbaum Associates. p.137-146.

[8] Lopes, D. (1996). Understanding Pictures. Oxford, Uk: Clarendon Press.

[13] Smith-Jackson, T. L. \& Wogalter, M. S. (2000). Application of cultural ergonomics to safety information research. In Proceedings of the 14th Triennial Conference of IEA/HFES. pp. $150-153$.

[12] Waarde, K.; Spinillo, C. G. (2011). Estudio de caso: el desarrollo de la información visual acerca de los medicamentos en Europa. In: J. Frascara (Org.). Qué es el diseño de información?. 1 ed. Buenos Aires: Ediciones Infinito, v. 1, p. 14-19. 